\title{
Deslocamento da oferta do MDF brasileiro no período de 2008-2017
}

\begin{abstract}
Desde meados de 2006 o MDF se destaca na competitividade de mercado, principalmente no Brasil e China, isso se deve ao baixo custo de produção e de matéria prima de florestas plantadas. A principal matéria-prima dos painéis brasileiros são provenientes das florestas plantadas de pinus e eucalipto, localizadas principalmente nas regiões sul e sudeste do país, e dos painéis produzidos no Chile, Espanha, EUA e Canadá, são os resíduos de madeira de outros processos industriais. $\mathrm{O}$ objetivo deste trabalho foi analisar a oferta do MDF brasileiro em um horizonte de dez anos. $\mathrm{O}$ estudo foi baseado nos dados de produção $\mathrm{e}$ exportação de painéis de MDF do Brasil, referente ao período de 2008 a 2017, disponível no site da Organização das Nações Unidas para Agricultura e Alimentação - FAO. O cálculo das taxas geométricas de crescimento foi realizado pelo software GRETL, no qual o tempo foi utilizado como regressor e os dados de preço e produção como variável dependente, sendo estabelecido estatisticamente, por regressão linear simples. Os deslocamentos da demanda e da oferta ocorrem em função de todos os fatores mercadológicos, exceto o preço, independentemente de serem conhecidos ou mensurados. Demonstrando quanto um determinado mercado estaria disposto a consumir ou ofertar a mais ou a menos de um produto sem alteração do preço. O comportamento do preço do MDF brasileiro no período analisado demonstrou uma taxa de crescimento negativa com -9,16. Verificou-se que em 2008 apresentou valor de U\$354,97/M³ e até 2011 apresentou uma valorização em seu preço, porém a partir de 2013 observou-se uma desvalorização do preço chegando a U\$ 211,19/M³ em 2017. Verificou-se aumento da quantidade produzida no período amostrado, combinado a queda de preço ambos significativos a $5 \%$ de probabilidade. A oferta do MDF brasileiro apresentou dessa forma deslocamento dominante para a direita no período de 2008 a 2017.
\end{abstract}

Palavras-chave: Economia Florestal; Painéis Aglomerados; Indústria Florestal; Florestas Plantadas.

\section{Displacement of the brazilian MDF supply in the period 2008-2017}

\begin{abstract}
Since mid-2006 the MDF stands out in the market competitiveness, mainly in Brazil and China, this is due to the low cost of production and raw material of planted forests. The main raw material of the Brazilian panels comes from pine and eucalypt plantation softwoods, mainly located in the south and southeast of the country, and the panels produced in Chile, Spain, USA and canada are the wood residues from other industrial processes. The objective of this work was to analyze the Brazilian MDF supply in a 10 year horizon. The study was based on the production and export data of MDF panels from Brazil, for the period from 2008 to 2017 , available on the website of the Food and Agriculture Organization of the United Nations (FAO). The calculation of the geometric growth rates was performed by the GRETL software, in which time was used as regressor and the price and production data as dependent variable, being established statistically by simple linear regression. The shifts in demand and supply occur because of all market factors, regardless of whether they are known or measured, except price. Demonstrating how much a market would be willing to consume or offer more or less of a product without price change. The behavior of the Brazilian MDF price in the analyzed period showed a negative growth rate with $-9,16$. In 2008, it had a value of US $\$ 354.97 / \mathrm{M}^{3}$ and until 2011 it had a price appreciation, but from 2013 onwards, a price depreciation of US $\$ 211.19 / \mathrm{M}^{3}$ was observed. 2017. There was an increase in the quantity produced in the sampled period, combined with a significant fall in prices to $5 \%$ probability. The Brazilian MDF showed a dominant shift of supply to the right in the period from 2008 to 2017.
\end{abstract}

Keywords: Forestry Economy; Particleboard; Forestry Industry; Plantation Softwoods.

Topic: Ciências Florestais

Reviewed anonymously in the process of blind peer.
Received: 12/04/2019

Approved: 27/05/2019
Douglas Valente de Oliveira (iD)

Universidade Federal do Oeste do Pará, Brasil

http://lattes.cnpq.br/8811200922396005

http://orcid.org/0000-0001-5773-1961

douglasvalenteoliveira@hotmail.com

\section{Rommel Noce (iD}

Universidade Federal do Oeste do Pará, Brasil http://lattes.cnpq.br/9915475270570854 http://orcid.org/0000-0001-8932-1297 noce.rommel@gmail.com

Juliana Mendes de Oliveira (DD

Universidade Federal do Oeste do Pará, Brasil http://lattes.cnpq.br/2689230773259816

http://orcid.org/0000-0003-3682-8208

julainameoli@yahoo.com.br

\author{
Laura Fernanda de Lima Lobato \\ Universidade Federal do Oeste do Pará, Brasil \\ http://lattes.cnpq.br/2689230773259816 \\ http://orcid.org/0000-0003-3682-8208 \\ laura.fll@hotmail.com \\ Silmara Ribeiro Leal ii \\ Universidade Federal do Oeste do Pará, Brasil \\ http://lattes.cnpq.br/6595393238585964 \\ http://orcid.org/0000-0003-4574-6693 \\ mribeiroleal@hotmail.com \\ Jobert Silva da Rocha (iD \\ Universidade Federal do Oeste do Pará, Brasil \\ http://lattes.cnpq.br/0097475774081856 \\ http://orcid.org/0000-0002-2370-3329 \\ jobert.job.rocha@gmail.com
}

\section{Referencing this:}

OLIVEIRA, D. V.; NOCE, R.; OLIVEIRA, J. M.; LOBATO, L. F. L.; LEAL, S. R.; ROCHA, J. S.. Deslocamento da oferta do MDF brasileiro no período de 2008-2017. Revista Ibero Americana de Ciências Ambientais, v.10, n.3, p.1-7, 2019. DOI: http://doi.org/10.6008/CBPC2179$\underline{6858.2019 .003 .0001}$

DOI: 10.6008/CBPC2179-6858.2019.003.0001 


\section{INTRODUÇÃO}

Os painéis de madeira surgiram através da demanda gerada pela escassez e encarecimento da madeira maciça que vem substituindo gradativamente. Além da necessidade de inovação da aplicação e do uso da madeira como matéria prima (EISFELD et al., 2012). Desde meados de 2006 o MDF se destaca na competitividade de mercado, principalmente no Brasil e China, isso se deve ao baixo custo de produção e de matéria prima de florestas plantadas (FAO, 2006).

O Ministério da Agricultura, Pecuária e Abastecimento (MAPA) em 2007 estimou que até 2020 o crescimento da produção de MDF no Brasil será de uma taxa um pouco menor que $4 \%$ a.a., sendo que a utilização de madeira de eucalipto e resíduos na produção aumentara sua participação (MAPA, 2007). Biazus et al. (2014) destacam que as vantagens dos painéis de madeira se devem a capacidade de aproveitamento integral das toras, possibilidade usar os resíduos, além de permitir a produção de painéis de grandes dimensões, em que o fator limitante é a dimensão das prensas e não das árvores.

Os principais demandantes de MDF no Brasil são a indústria moveleira, que é atendida diretamente (54\%) ou por meio de revendedores (31\%), e a indústria da construção civil (8\%) (BNDES, 2008). A base do setor de painéis de fibras de média densidade (MDF), as florestas plantadas, têm grande competitividade no Brasil em comparação ao resto do mundo em função das condições edafoclimáticas e fundiárias, à verticalização do setor e a qualidade da mão de obra aplicada (VIDAL et al., 2011).

A principal matéria-prima dos painéis brasileiros são provenientes das florestas plantadas de pinus e eucalipto, localizadas principalmente nas regiões sul e sudeste do país, e dos painéis produzidos no Chile, Espanha, EUA e Canadá, são os resíduos de madeira de outros processos industriais. Logo, os painéis que são produzidos no exterior apresentam vantagem em comparação aos painéis no Brasil, visto que utilizam resíduos para fabricar seus painéis, assim minimizando impactos ambientais na produção agrícola (FREIRE et al., 2015).

De acordo com Almeida et al. (2010) a formação do preço da madeira que é insumo para produção de MDF depende, principalmente, dos custos de produção, mas também é o mercado quem determina ou estabelece esse preço, que é resultante do equilíbrio entre as forças de oferta e demanda. Portanto, tanto as variáveis determinantes da oferta quanto da demanda de madeira devem ser consideradas na especificação de um modelo explicativo de preço.

Quando o preço de determinada madeira atinge o nível em que a quantidade ofertada é igual à quantidade demandada então alcançasse o equilíbrio de mercado (MANKIW, 2013). Diante do exposto verifica-se a necessidade de estudos econométricos voltados para a indústria florestal brasileira, logo, o objetivo deste trabalho foi analisar a oferta do MDF brasileiro em um horizonte de dez anos, para estimar o deslocamento da curva de oferta do MDF. 


\section{METODOLOGIA}

O estudo foi baseado nos dados de produção e exportação de painéis de MDF do Brasil, referente ao período de 2008 a 2017, disponível no site da Organização das Nações Unidas para Agricultura e Alimentação - FAO. Após corrigir e filtrar os dados, foram estruturados em séries históricas no Microsoft Excel, o preço (valor da exportação/quantidade da exportação) e a produção (Volume em milhão de $\mathrm{m}^{3}$ ) para calcular a taxa geométrica de crescimento. O cálculo das taxas geométricas de crescimento foi realizado pelo software GRETL, no qual o tempo foi utilizado como regressor e os dados de preço e produção como variável dependente, sendo estabelecido estatisticamente, por regressão linear simples, através da seguinte expressão da equação 1.

$$
T G C=(\text { Ant } \log \beta-1) \times 100 \quad(\text { Equação 1) }
$$

Sendo:

TCG = Taxa geométrica de crescimento;

$\beta=$ Coeficiente de regressão.

O coeficiente de regressão teve seus valores estimados com o ajuste da equação de tendência (Equação 2), através da série histórica das variáveis dependentes.

$$
\log Y=a+\beta T
$$

(Equação 2)

Onde:
$\mathrm{Y}=$ variável;
$\mathrm{a}=$ constante da regressão; e
$\mathrm{T}=$ tempo

A partir das taxas de crescimento calculadas, foi determinado o comportamento o comportamento de mercado do MDF, explicado, principalmente, por fatores ligados à oferta ou à demanda. Neste trabalho foi admitido a produção com oferta e os deslocamentos ao longo do tempo e das curvas de oferta e demanda (MANKIW, 2013). A metodologia proposta por Almeida et al. (2009) avalia o efeito do deslocamento da demanda e oferta, no qual um aumento ou queda do preço e da quantidade produzida do bem em questão apresentarão tendências específicas (Tabela 1).

Os deslocamentos da demanda e da oferta ocorrem em função de todos os fatores mercadológicos, independentemente de serem conhecidos ou mensurados, exceto o preço. Demonstrando quanto um determinado mercado estaria disposto a consumir ou ofertar a mais ou a menos de um produto sem alteração do preço. Assim, quando a curva de demanda desloca para a direita o mercado estará disposto a consumir uma quantidade maior do produto ao mesmo preço. Já quando desloca para a esquerda os consumidores estariam dispostos a retirar do mercado uma quantidade menor ao mesmo preço. A disposição dos produtores em ofertar um determinado produto no mercado ocorre de forma análoga. Sendo o equilíbrio determinado pela demanda e oferta, o deslocamento de ambas permite perscrutar a dinâmica e as condições de equilíbrio do mercado (MANKIW, 2013).

Foram admitidos aumentos tanto para o preço como para a produção quando as TGC's se mostraram positivas e de forma análoga, quando negativas admitiu-se quedas seja no preço ou na produção. 0 deslocamento dominante da oferta para a direita pressupõe sentidos inversos da quantidade e do preço. Sendo um aumento da produção e uma queda do preço. O deslocamento dominante da oferta para esquerda 
levaria a reduções tanto da quantidade como dos preços. O deslocamento da demanda para a direita pressupõe aumentos de quantidade e preços enquanto seu deslocamento para a direita implicaria em redução das quantidades com preços mais elevados (ALMEIDA et al., 2009). Os resultados foram observados através da taxa geométrica de crescimento e posteriormente foi construído os gráficos da evolução da produção e preço de painéis.

Tabela 1: Deslocamentos de oferta e demanda.

\begin{tabular}{|c|c|c|}
\hline Tendência & Sinal TGC's & Deslocamentos \\
\hline$\uparrow D$ & $\begin{array}{l}\text { (+) Produção } \\
(+) \text { Preço }\end{array}$ & Demanda para a direta \\
\hline$\downarrow D$ & $\begin{array}{l}\text { (-) Produção } \\
(+) \text { Preço }\end{array}$ & Demanda para a esquerda \\
\hline 个० & $\begin{array}{l}\text { (+) Produção } \\
\text { (-) Preço }\end{array}$ & Oferta para a direta \\
\hline$\downarrow 0$ & $\begin{array}{l}\text { (-) Produção } \\
\text { (-) Preço }\end{array}$ & Oferta para a esquerda \\
\hline
\end{tabular}

Fonte: Almeida et al. (2009).

\section{RESULTADOS E DISCUSSÃO}

O comportamento do preço do MDF brasileiro no período analisado demonstrou uma taxa de crescimento negativa com -9,16. Verifica-se que em 2008 apresentava valor de U\$ 354,97/M³ e até 2011 apresentou uma valorização em seu preço, porém a partir de 2013 observa-se uma desvalorização chegando a valer U\$ 211,19/M³ em 2017 (Figura 1). Observou-se entre 2002 e 2008 crescimento de preço, sendo o reajuste de 12, 7 \% a.a. apesar da crise financeira em 2009, com retração da demanda local e queda no preço praticado. Os painéis de MDF apresentaram um reajuste de $8 \%$ em outubro de 2009 e $7 \%$ em janeiro de 2010 (BIAZUS et al., 2014).

Diferentemente do comportamento observado no preço, a produção de MDF no período analisado apresentou uma taxa geométrica de crescimento positiva com 10,38. A produção brasileira de MDF, entre 2008 a 2017, passou de 1,8 milhão de $\mathrm{m}^{3}$ para 4 milhões de $\mathrm{m}^{3}$, aumentando mais de $100 \%$. A produção manteve-se crescente até 2015 , onde atingiu o máximo, com 4,4 milhões de $\mathrm{m}^{3}$ de painéis produzidos, no ano posterior houve um decréscimo na produção de aproximadamente $380 \mathrm{mil} \mathrm{m}^{3}$ (Figura 2).

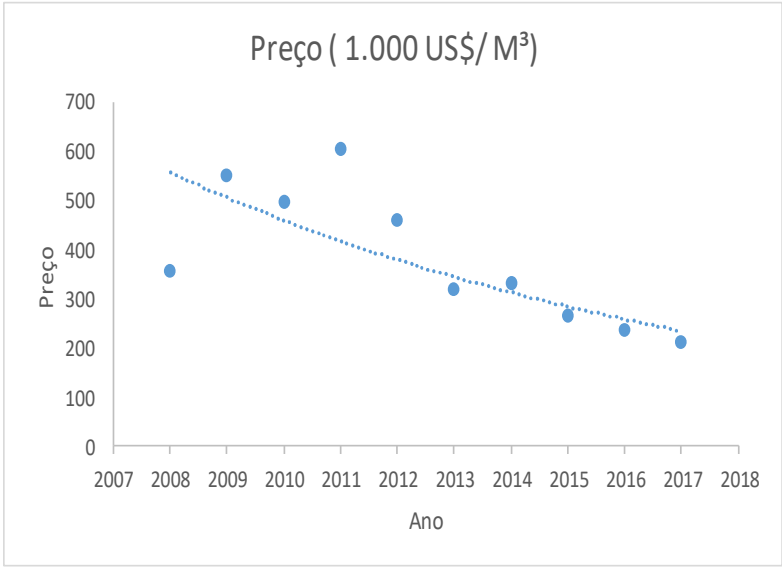

Figura 1: Evolução do preço do MDF brasileiro.

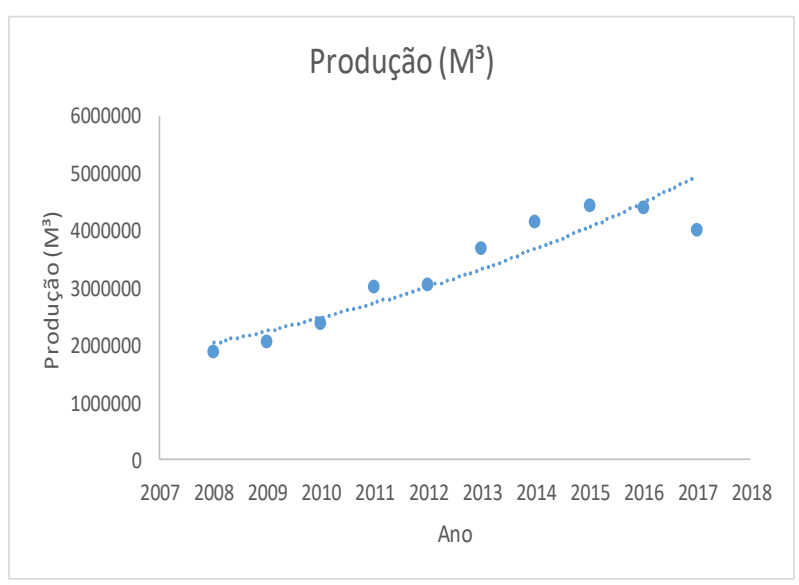

Figura 2: Evolução da produção do MDF brasileiro. 
Por sua vez, o consumo nacional de MDF, evoluiu de 2,0 milhões de $\mathrm{m}^{3}$, em 2006 para 3,8 milhões de $\mathrm{m}^{3}$, em 2016, apresentando um incremento de $84,8 \%$. Chegando a superar a produção no ano de 2011, justificando as maiores taxas registradas de importações de MDF neste período para suprir a demanda interna. Logo após 2012 o consumo interno reduziu em comparação aos anos anteriores, favorecendo o incremento das exportações (ARAUJO et al., 2018). Para Radichi (2004) mesmo quando há um cenário pouco favorável à exportação, a agroindústria que abrange diversos setores, como também o florestal, consegue se manter como um dos setores mais eficientes e produtivos do país, e isso se deve a seu alto nível tecnológico e sua grande competitividade externa.

Verificou-se aumento da quantidade produzida no período amostrado, combinado a queda de preço ambos significativos a $5 \%$ de probabilidade. Portanto pode-se admitir deslocamento dominante da oferta para direita. O que corrobora os resultados de Salles et al. (2016) ao verificar a dinâmica de preço e quantidades exportadas de produtos florestais brasileiros no período de 1995 a 2013.

O crescimento na produção de painéis no Brasil, traz consequências como o aumento no impacto ambiental, seja devido à necessidade de madeira ou durante o processo de produção. No entanto, é importante frisar que pesquisadores no Brasil vem desenvolvendo a aplicação de resíduos agroindustriais, como a casca de coco verde na produção de painéis aglomerados (CRAVO et al., 2015).

Para Rowell et al. (2000) os painéis podem ser produzidos através de qualquer material lignocelulósico que lhe confiram qualidade igual ao produzido a partir de madeira, pois a composição química de materiais lignocelulósico são semelhantes ao de madeira dura, que contém teor de lignina e hemicelulose desejáveis.

Entretanto trabalhos realizados com materiais como casca de café (MENDES et al., 2010), casca de amendoim (GULER et al., 2017) e caule de girassol (BEKTAS et al., 2005), denotam efeitos negativos nas propriedades físico-mecânicas dos painéis. Porém o uso de fibra de coco (COLLI et al., 2010) e fibra da casca do coco-verde combinada a resina poliuretana bi componente à base de óleo de mamona (FIORELLI et al., 2012) contribuem para melhorar o desempenho físico-mecânico dos painéis.

O bom desempenho nas exportações de commodities florestais brasileiras geralmente ocorre devido a determinadas vantagens. Atreladas principalmente a fatores endógenos, como por exemplo baixo custo de produção e taxa de câmbio favorável. A desvalorização do câmbio brasileiro, e a alta produtividade das florestas são fatores determinantes no sucesso do setor florestal no mercado internacional (VALVERDE et al., 2004).

Para Gomes (2014), o setor florestal brasileiro enfrenta grandes desafios, como o câmbio desfavorável, a crise nos países importadores, altas barreiras comerciais e o custo Brasil. A crise nos países que importam produtos florestais afeta muito o mercado no Brasil, devido a sua vulnerabilidade, que facilmente pode ser verificada na crise que ocorreu em 2008 e 2009. Quando ocorreram consequências negativas ao setor. Denotando a necessidade de ajustes que reflitam em custos com infraestrutura, burocracia, processos onerosos de licenciamento ambiental além de elevadas taxas de juros e cargas 
tributarias. O que encarece os insumos e produtos brasileiros em comparação aos estrangeiros (SALLES et al., 2016).

O comportamento das exportações de painéis de madeira tem ligação direta com o maior importador, os Estados Unidos. Em 2005, houve um aquecimento do mercado imobiliário no EUA, sendo que o ápice desse aquecimento ocorreu em junho de 2005, sendo solicitadas 211,9 mil permissões para construção. O Brasil destinava aos Estados Unidos 34\% das suas exportações de painéis de madeira neste ano. A desvalorização do real frente ao dólar no período diminuiu os preços de produtos como o MDF, pois o comércio internacional de commodities tem por base a moeda norte americana. A tendência nas quedas dos preços acompanhou também a desvalorização do real em 2009, como consequência da crise mundial (SALLES et al., 2016) (UNITED STATES CENSUS BUREAU, 2015).

Tanto a oferta como a demanda dos painéis de fibras, como o MDF brasileiro, estão diretamente ligadas as exportações mundiais, sendo essas complementares à expansão das atividades globais e baseadas nas condições reais de competitividade da indústria nacional. Sendo necessário direcionar esforços para aumentar as vantagens de produção de escala, preço e promoção do produto brasileiro no mercado internacional (BRASIL et al., 2003).

\section{CONCLUSÕES}

Conclui-se que o MDF brasileiro apresentou um deslocamento dominante da oferta para a direita no período de 2008 a 2017. Motivada pela penetração de mercado proporcionada ao MDF nacional em função da sensível redução de preço frente aos concorrentes internacionais decorrente da relação cambial. Associada ainda a incrementos no consumo da principal nação importadora.

\section{REFERÊNCIAS}

ALMEIDA, A. N.; SANTOS, A. J.; SILVA, J. C. G. L. D.; BITTENCOURT, A. M.. Análise do mercado dos principais produtos não-madeiráveis do estado do Paraná. Floresta, Curitiba, v.39, n.4, p.753-763, 2009. DOI:

http://dx.doi.org/10.5380/rf.v39i4.16310

ALMEIDA, A. N.; SILVA, J. C. G. L.; ANGELO, H.; NUÑEZ, B. E. C.. Análise dos fatores que influenciam o preço da madeira em tora para processamento mecânico no Paraná. Cerne, Lavras, v.16, n.2, p.243-250, 2010. DOI: http://dx.doi.org/10.1590/S0104-77602010000200017

ARAUJO, E. S.; VIÉGAS, D. R.; SOUSA, T. B.; SOUZA, S. G.; SILVA, J. C.; REIS, V. N. O.; SOUSA, I. A. L.; MORI, F. A.. Evolução do mercado nacional de painéis MDF e MDP. In: ENCONTRO BRASILEIRO EM MADEIRAS E EM ESTRUTURAS DE MADEIRA, 16. Anais. São Carlos, 2018. p.1-9.

BEKTAS, I.; GULER, C.; KALAYCIOĞLU, H.; MENGELOGLU, F.; NACAR, M.. The manufacture of particleboards using sunflower stalks (Helianthus annuus L.) and poplar wood (Populus alba L.). J. Compos. Mater, Lancaster, v.39, n.5, p.467-473, 2005. DOI:

http://doi.org/10.1177\%2F0021998305047098
BIAZUS, A.; HORA, A. B.; LEITE, B. G. P.. Panorama de mercado: painéis de madeira. Rio de Janeiro: BNDES Setorial, 2014.

BNDES. Setorial: Painéis de madeira no Brasil: panorama e perspectivas. Rio de Janeiro, 2008.

BRASIL, A. A.; ANGELO, H.; SANTOS, A. J.; BERGER, R.; SILVA, J. C. G. L.. Demanda de exportação de painéis de madeira do Brasil. Floresta, Curitiba, v.33, n.2, p.135-146, 2003. DOI: http://dx.doi.org/10.5380/rf.v33i2.2268

MAPA. Ministério da Agricultura, Pecuária e Abastecimento. Cadeia produtiva de madeira. Secretaria de Política Agrícola. Brasília: IICA, 2007.

CRAVO, J. C. M.; SARTORI, D. L.; FIORELLI, J.; BALIEIRO, J. C. C.; SAVASTANO JR, H.. Painel aglomerado de resíduos agroindustriais. Ciência Florestal, Santa Maria, v.25, n.3, p.721-730, 2015. DOI: http://dx.doi.org/10.5902/1980509819675

COLLI, A.; VITAL, B. R.; CARNEIRO, A. C. O.; SILVA, J. C.; CARVALHO, A. M. M. L.; LUCIA, R. M. D.. Propriedades de chapas fabricadas com partículas de madeira de paricá 
(Schyzolobium amazonicum) e fibra de coco verde (Cocos nucifera). Revista Árvore, Viçosa, v.34, n.2, p.333-338, 2010. DOI: http://dx.doi.org/10.1590/S0100-67622010000200016

EISFELD, C. L.; BERGER, R.. Análise das Estruturas de Mercado das Indústrias de Painéis de Madeira (Compensado, MDF e OSB) no Estado do Paraná. Floresta, Curitiba, v.42, n.1, p.21-34, 2012. DOI:

http://dx.doi.org/10.5380/rf.v42i1.26289

FIORELLI, J.; CURTOLO, D. D.; BARRERO, N. G.; SAVASTANO JUNIOR, H.; PALLONE, E. M. J. A.; JOHNSON, R.. Particulate composite based on coconut fiber and castor oil polyurethane adhesive: An ecoefficient product. Industrial Crops and Products, v.40, p.69-75, 2012. DOI: http://doi:10.1016/j.indcrop.2012.02.03

FREIRE, A. L. F.; FIGUEIRÊDO, M. C. B.; ROSA, M. F.; ARAUJO JUNIOR, C. P.. Impactos ambientais de painéis de madeira e derivados: uma revisão de literatura. Espacios, Caracas, v.6, n.10, p.1-12, 2015.

FAO. Tendencias y perspectivas del sector forestal en América Latina y el Caribe. Roma: Estudio FAO Montes, 2006.

GULER, C.; COPUR, Y.; TASCIOGLU, C.. The manufacture of particleboards using mixture of peanut (Arachis hypoqaea) and European Black pine (Pinus nigra Arnold) wood chips. Bioressource Technology, New York, v. 99, p. 2893-2897, 2007. DOI: http://doi:10.1016/j.biortech.2007.06.013
MANKIW, N. G.. Introdução à economia. 5 ed. Cambridge: Universidade de Harvard, 2013.

SALLES, T. T.; ISBAEX, C.; SILVA, M. L; VALVERDE, S. R.; LUZ, T. M. O.. Dinâmica de preços e quantidades exportadas de produtos florestais brasileiros, 1995-2013. Pesquisa Florestal Brasileira, Colombo, v.36, n.88, p.451-457, 2016. DOI: http://doi:10.4336/2016.pfb.36.88.1049

RADICCHI, C. C.. Competitividade das exportações brasileiras de celulose: uma análise do custo Brasil. Dissertação (Mestrado em Economia Aplicada) Universidade Federal de Viçosa, Viçosa, 2004.

ROWELL R. M.; HAN J. S.; ROWELL J. S.. Characterization and Factors Affecting Fiber Properties. In: NATURAL POLYMERS AND AGROFIBERS BASED COMPOSITES. Anais. São Carlos: Embrapa Instrumentação Agropecuária, 2000.

UNITED STATES CENSUS BUREAU. Population estimates. Washington, 2015.

VALVERDE, S. R.; SOARES, N. S.; SILVA, M. L.; JACOVINE, L. A. G.; NEVIRA, S. A.. O comportamento do mercado da madeira de eucalipto no Brasil. Biomassa \& Energia, Viçosa, v.1, n.4, p.393-403, 2004.

VIDAL, A.; HORA, A.. A atuação do BNDES nos setores de florestas plantadas, painéis de madeira, celulose e papéis: o período 2001-2010. BNDES Setorial, 2011.

A CBPC - Companhia Brasileira de Produção Científica (CNPJ: 11.221.422/0001-03) detém os direitos materiais desta publicação. Os direitos referem-se à publicação do trabalho em qualquer parte do mundo, incluindo os direitos às renovaç̃ões, expansões e disseminações da contribuiç̃o, bem como outros direitos subsidiários. Todos os trabalhos publicados eletronicamente poderão posteriormente ser publicados em coletâneas impressas sob coordenação da Sustenere Publishing, da Companhia Brasileira de Produção Científica e seus parceiros autorizados. Os (as) autores (as) preservam os direitos autorais, mas não têm permissão para a publicação da contribuição em outro meio, impresso ou digital, em português ou em tradução. 